\title{
Development of a simple nonalcoholic fatty liver disease scoring system indicative of metabolic risks and insulin resistance
}

\author{
Seogsong Jeong ${ }^{1}$, Kyuwoong Kim ${ }^{2}$, Jooyoung Chang ${ }^{1}$, Seulggie Choi ${ }^{1}$, Sung Min Kim ${ }^{1}$, Joung Sik Son ${ }^{3}$, \\ Gyeongsil Lee ${ }^{3}$, Won Kim ${ }^{4}$, Sang Min Park ${ }^{1,3}$ \\ ${ }^{1}$ Department of Biomedical Sciences, Seoul National University Graduate School, Seoul, Republic of Korea; ${ }^{2}$ National Cancer Control Institute, \\ National Cancer Center, Goyang-si, Gyeonggi-do, Republic of Korea; ${ }^{3}$ Department of Family Medicine, Seoul National University Hospital, \\ Seoul, Republic of Korea; ${ }^{4}$ Division of Gastroenterology and Hepatology, Department of Internal Medicine, Seoul Metropolitan Government Seoul \\ National University Boramae Medical Center, Seoul, Republic of Korea \\ Contributions: (I) Conception and design: All authors; (II) Administrative support: All authors; (III) Provision of study materials or patients: All \\ authors; (IV) Collection and assembly of data: S Jeong, K Kim, SM Park; (V) Data analysis and interpretation: S Jeong, K Kim, SM Park; (VI) \\ Manuscript writing: All authors; (VII) Final approval of manuscript: All authors. \\ Correspondence to: Sang Min Park, MD, PhD, MPH. Department of Biomedical Sciences, Seoul National University College of Medicine, 101 \\ Daehak-ro, Jongno-gu, Seoul 03080, Republic of Korea. Email: smpark.snuh@gmail.com.
}

Background: Nonalcoholic fatty liver disease (NAFLD) is estimated to affect approximately $30 \%$ population worldwide. However, there is yet a basic and generally implementable approach to define individuals at risk for NAFLD estimative of metabolic risk.

Methods: Total of 3,634 general participants without history of liver disease and alcohol consumption who received the Korean National Health and Nutrition Examination Survey between 2008 and 2010 were studied. Logistic regression was used to identify significant covariates indicative of NAFLD. Multivariableadjusted logistic regression was carried out for evaluation on estimative impact of the derived score on metabolic risks.

Results: Sex [female; odd ratio (OR), 2.492; 95\% confidence interval (CI), 1.921-3.233; $\mathrm{P}<0.001$ ], waist circumference (WC) (OR, 1.093; 95\% CI, 1.077-1.110, $\mathrm{P}<0.001)$ systolic blood pressure (OR, 1.033; 95\% CI, 1.025-1.040; $\mathrm{P}<0.001)$, fasting serum glucose (FSG) (OR, 1.032; 95\% CI, 1.026-1.038; $\mathrm{P}<0.001$ ), triglyceride (OR, 1.007; 95\% CI, 1.006-1.009; $\mathrm{P}<0.001$ ), and alanine aminotransferase (OR, 1.110; 95\% CI, 1.097-1.124; $\mathrm{P}<0.001)$ were independently indicative of NAFLD, which constituted the derived scoring system. The system revealed a significant correlation with the NAFLD liver fat score, fatty liver index (FLI), body mass index, age, alanine aminotransferase, and triglycerides (BAAT) score, lipid accumulation product, and hepatic steatosis index. In addition, the derived score was significantly estimative of insulin resistance.

Conclusions: The developed score may be supportive of stratification of individuals at high risk of NAFLD, non-invasive fast diagnosis of NAFLD, and estimation of metabolic risk. Future studies that compare the derived score with standard diagnostic tests-validated data, such as ultrasonography of the liver, are needed.

Keywords: Nonalcoholic fatty liver disease (NAFLD); non-invasive diagnosis; metabolic risk factors; insulin resistance

Submitted Mar 29, 2020. Accepted for publication Aug 28, 2020.

doi: 10.21037/atm-20-2951

View this article at: http://dx.doi.org/10.21037/atm-20-2951 


\section{Introduction}

Nonalcoholic fatty liver disease (NAFLD) is currently being considered a major cause of chronic liver diseases worldwide that affect approximately $30 \%$ of general population in most countries, including the United States and South Korea $(1,2)$. In the United States, NAFLD is projected to expand from 83.1 million in 2015 to 100.9 million in 2030 (3). This rising trend will lead to an increased burden of nonalcoholic steatohepatitis (NASH) and advanced fibrosis, culminating in hepatocellular carcinoma (HCC) and end-stage liver disease (4). Compared to other etiologic factors of HCC, NAFLDassociated HCC occasionally occurs without cirrhotic liver when routine screening for HCC is yet conducted, thus presenting with more advanced stage and less amenable to curative approaches $(5,6)$.

NAFLD has been regarded as the liver manifestation of the metabolic syndrome by some researchers considering its close associations with obesity, insulin resistance, hypertension, and dyslipidemia (7). Boza et al. (8) showed that NASH is present in more than $60 \%$ of patients with obesity undergoing gastric bypass surgery as found by histological examination, and suggested that insulin resistance is highly predictive of NASH. In addition, it was noticed that NASH also enhances insulin resistance leading to a vicious cycle, supporting close associations between NAFLD, metabolic syndrome, obesity, and insulin resistance (9).

Liver biopsy is considered reliable and sometimes essential for diagnosis of NAFLD by grading distinctive histological patterns, including steatosis, hepatocellular ballooning, lobular inflammation, and fibrosis (10). However, it is unpractical to perform liver biopsy for all population at risk for NAFLD due to its invasiveness and cost-effectiveness (11). Therefore, there has been an increasing interest in alternative diagnostic strategies for NAFLD using non-invasive assessments, such as transient elastography (12). In addition, further simplified methods without imaging approaches for application in a general population are increasingly required.

In 2009, NAFLD liver fat score, consisting of the metabolic syndrome, type 2 diabetes, fasting serum insulin (FSI), aspartate aminotransferase (AST), and AST-to-alanine aminotransferase (ALT) ratio, was derived and validated to be highly effective with 0.87 and 0.86 area under curve (AUC) values, respectively (13). They also confirmed that addition of genetic factors could only improve $<1 \%$ of predictive accuracy. Although this score is reliable and easy to use, the inclusion of FSI limits its application in a number of hospitals and health check centers in terms of data availability and cost-effectiveness. Besides, the impact of non-invasive NAFLD scoring systems on the estimation of metabolic risks, including metabolic syndrome, obesity, and insulin resistance remains to be confirmed.

Herein, we conducted this study to develop a simplified NAFLD score applicable to general population and health examination datasets composed of demographic and serologic characteristics, compared with preexisting scoring systems for estimation of NAFLD, including the NAFLD liver fat score, fatty liver index (FLI), body mass index, age, alanine aminotransferase, and triglycerides (BAAT) score, lipid accumulation product (LAP), and hepatic steatosis index (HSI), and testified its impact on the estimation of metabolic syndrome, metabolic risk factors, obesity, and the homeostatic model assessment for insulin resistance (HOMA-IR) in the Korean National Health and Nutrition Examination Survey (KNHANES) dataset (14-17). We present the following article in accordance with the STROBE reporting checklist. available at: http://dx.doi. org/10.21037/atm-20-2951.

\section{Methods}

\section{Study population}

The KNHANES (http://knhanes.cdc.go.kr) dataset, which is a nationally representative cross-sectional surveillance system that has been evaluating the health and nutritional status of noninstitutionalized Korean civilians residing in Korea since 1998, was used in the present study (18). Due to unavailability of a diagnostic test results within the dataset, the NAFLD liver fat score, which is derived and validated using proton magnetic resonance spectroscopy, was used to operationally define NAFLD for the analyses (13). Based on availability of FSI, which is involved in the NAFLD liver fat score, participants who received examination between 2008 and 2010 were studied. From 29,235 participants, 5,816 adult participants were eligible for the NAFLD liver fat score, FLI, BAAT score, and LAP evaluations. Among them, participants with history of liver diseases, including hepatitis virus infection, liver cirrhosis, and cancer $(n=137)$. Those with alcohol consumption $(n=2,045)$, defined as more than once per month of frequency, were further excluded to rule out participants with potential liver injury induced by alcohol consumption. Finally, a total of 3,634 
participants were included in this study (Figure S1). All participants of the KNHANES provided informed consent before the survey. The study was conducted in accordance with the Declaration of Helsinki (as revised in 2013). No approval from the Institutional Review Board was needed as KNHANES is publicly available from the Korea Centers for Disease Control and Prevention (https://www.cdc. go.kr).

\section{Data collection}

The dataset is composed of health interview, health examination, and nutrition survey. Nutrition survey was carried out a week after health interview and health examination surveys, which were performed by trained medical staff and interviewers. Weight and height measurements were performed without socks or accessories. Body mass index (BMI) was calculated by dividing weight $(\mathrm{kg})$ by height ${ }^{2}\left(\mathrm{~m}^{2}\right)$. Obesity was defined as BMI $\geq 25 \mathrm{~kg} / \mathrm{m}^{2}$ according to the Asia-Pacific criteria (19). Waist circumference (WC) was measured from the mid-axillary line to the lower rib. Metabolic syndrome was defined when three or more of the following criteria are met: WC $>102 \mathrm{~cm}$ for men and $>88 \mathrm{~cm}$ women, systolic blood pressure $\geq 130$ or diastolic blood pressure $\geq 85 \mathrm{mmHg}$, fasting triglyceride (TG) $>150 \mathrm{mg} / \mathrm{dL}$, fasting high-density lipoprotein cholesterol (HDL) $<40 \mathrm{mg} / \mathrm{dL}$ for men or $<50 \mathrm{mg} / \mathrm{dL}$ for women, and fasting serum glucose (FSG) $>100 \mathrm{mg} / \mathrm{dL}$ according to the National Cholesterol Education Program-Adult Treatment Panel III (20).

\section{Scoring system}

The NAFLD liver fat score was calculated by $1.18 \times$ metabolic syndrome $+0.45 \times$ diabetes $(2$, if yes; 0 , if no) $+0.15 \times$ FSI $(\mathrm{mU} / \mathrm{L})+0.04 \times$ AST $(\mathrm{U} / \mathrm{L})-0.94 \times(\mathrm{AST} /$ ALT) -2.89 (13). The FLI was calculated by $\left[\mathrm{e}^{0.953} \times \log _{\mathrm{e}}{ }^{(\mathrm{TG})}\right.$ $+0.139 \times \mathrm{BMI}+0.718 \times \log _{\mathrm{e}}^{(\gamma-\text { glutanyyl transspeptiduse } ;-G T)}+0.053 \times \mathrm{WC}$ $-15.745] /\left[1+\mathrm{e}^{0.953} \times \log _{\mathrm{e}}{ }^{(\mathrm{TG})}+0.139 \times \mathrm{BMI}+0.718 \times \log _{\mathrm{e}}^{(\gamma-G T)}\right.$ $+0.053 \times$ WC -15.745$] \times 100$ (14). BAAT score was calculated by the sum of the followings: $B M I \geq 28$ ( 1 point), Age $\geq 50$ years ( 1 point), ALT $\geq 2 \mathrm{~N}$ ( 1 point), and $T G$ $\geq 1.7 \mathrm{mmol} / \mathrm{L}$ (1 point) (15). LAP was calculated by (WC $-65) \times$ TG if men; (WC -58$) \times$ TG if women (16). The HSI was calculated by ALT/AST ratio $\times 8+$ BMI $(+2$ for female; +2 for diabetes mellitus) (17). The cut-off values for NAFLD were $>-0.640,<30$, and $\geq 36$ for the NALFD liver fat score, FLI, and HSI, respectively. In addition, the
HOMA-IR was calculated by FSG $\times$ FSI/405 (21).

\section{Statistical analysis}

All statistical analyses were performed using SAS version 9.4 (SAS Institute Inc.) and R Project for Statistical Computing (https://www.r-project.org/). Continuous variables and categorical variables were presented as mean [standard deviation (SD)] and number (\%), respectively. All values were rounded to three decimal places. Univariable and multivariable analyses were performed using the logistic regression model, which were presented with odd ratio (OR), 95\% confidence interval (CI), and concordance index (C-index). For evaluation on the effects of K-NAFLD score on metabolic risk, insulin resistance, and obesity, the multivariable model was adjusted for age, household income, and lifestyle factors, including smoking status and strength exercise. The cut-off values for highly indicative and moderately indicative $\mathrm{C}$-indices were defined to $>0.8$ and $>0.6$, respectively. Correlations between factors in for each section were evaluated using the spearman correlation test. The cut-off values for NAFLD by the developed model were defined by the $95 \%$ of mean score. Pearson's correlation test with scatter matrix was applied for visualization of correlations between the scoring systems. Correlations between the developed model and metabolic risk factors were described using the scatter plot with $95 \%$ predictive ellipse. Receiver operating curves (ROC) with AUC values were generated for sensitivity and specificity evaluation. The cases with missing variables were excluded from the analysis. $\mathrm{P}<0.05$ was considered statistically significant.

\section{Results}

\section{Participant characteristics}

There were a total of 3,634 participants with a mean age of 46.8 years and a female proportion of $62.5 \%$ (Table 1 ). The mean BMI and WC were $23.5 \mathrm{~kg} / \mathrm{m}^{2}\left(\mathrm{SD}, 3.3 \mathrm{~kg} / \mathrm{m}^{2}\right)$ and $80.0 \mathrm{~cm}(\mathrm{SD}, 10.0 \mathrm{~cm})$, respectively. The average blood pressure was within the normal range. The mean FSG, FSI, total cholesterol, HDL, TG, and low-density lipoprotein cholesterol (LDL) were $95.9 \mathrm{mg} / \mathrm{dL}, 10.5 \mu \mathrm{IU} / \mathrm{mL}$, $187.1 \mathrm{mg} / \mathrm{dL}, 52.4 \mathrm{mg} / \mathrm{dL}, 120.7 \mathrm{mg} / \mathrm{dL}$, and $112.8 \mathrm{mg} / \mathrm{dL}$, respectively. The liver function test showed $20.9 \mathrm{IU} / \mathrm{L}$, $20.3 \mathrm{IU} / \mathrm{L}$, and 26.0 IU/L for mean AST, ALT, and $\gamma$-GT. Regarding SD for $\gamma$-GT that was larger than the mean 
Table 1 Baseline characteristics of the participants

\begin{tabular}{lc}
\hline Characteristic & $\begin{array}{c}\text { Participant } \\
(\mathrm{n}=3,634)\end{array}$ \\
\hline Age, years & $46.8(15.5)$ \\
Sex, female, $\mathrm{n}(\%)$ & $2,273(62.5)$ \\
Height, cm & $162.2(8.9)$ \\
Weight, kg & $61.9(11.3)$ \\
Body mass index, kg/m ${ }^{2}$ & $23.5(3.3)$ \\
Waist circumference, cm & $80.0(10.0)$ \\
Systolic blood pressure, mmHg & $118.7(17.4)$ \\
Diastolic blood pressure, mmHg & $76.2(10.5)$ \\
Fasting serum glucose, mg/dL & $95.9(20.9)$ \\
Glycosylated hemoglobin, type A1C, \% & $7.4(1.6)$ \\
Fasting serum insulin, $\mu \mathrm{IU} / \mathrm{mL}$ & $10.5(5.5)$ \\
Total cholesterol, mg/dL & $187.1(36.0)$ \\
High-density lipoprotein cholesterol, mg/dL & $52.4(12.6)$ \\
Triglyceride, mg/dL & $120.7(83.1)$ \\
Low-density lipoprotein cholesterol, mg/dL & $112.8(31.4)$ \\
Aspartate aminotransferase, IU/L & $20.9(9.4)$ \\
Alanine aminotransferase, IU/L & $20.3(15.6)$ \\
Blood urea nitrogen, mg/dL & $26.0(29.2)$ \\
\hline Creatinine, mg/dL & $13.7(4.5)$ \\
\hline
\end{tabular}

Data are mean (standard deviation) unless indicated otherwise.

value, the median value was also calculated, which was 18.0 IU/L (interquartile range, 14.0-28.0). In the kidney function test, $13.7 \mathrm{mg} / \mathrm{dL}$ and $0.8 \mathrm{mg} / \mathrm{dL}$ of mean values were found for blood urea nitrogen and creatinine.

\section{Univariable analysis for identification of significantly indicative factors for NAFLD}

Twenty-four factors from 8 distinctive sections defined in the KNHANES, including demographic, measurement, blood pressure, diabetes mellitus test, dyslipidemia test, liver function test, kidney function test, and urine test, were evaluated in univariable analyses (Table 2). Results indicated that the involved factors are generally and significantly reflective of NAFLD, except for glycosylated hemoglobin type $\mathrm{A} 1 \mathrm{C}(\mathrm{P}=0.465)$. The highest $\mathrm{C}$-index was found in
FSI (0.846), followed by ALT (0.833), WC (0.818), $\gamma$-GT (0.796), BMI (0.789), TG (0.777), FSG (0.776), AST (0.751), weight $(0.740)$, systolic blood pressure $(0.733)$, HDL $(0.717)$, diastolic blood pressure (0.705), age (0.635), LDL (0.610), and total cholesterol (0.601).

\section{Quality control for covariate selection}

In quality control, the following 3 terms were considered: (I) generalizability assessed by data availability at general health examination cohorts along with cost-effectiveness, (II) correlation assessed by the Spearman correlation test for evaluation of representativeness for other factors included within the same section, and (III) accuracy evaluated by $\mathrm{C}$-index from univariable analyses. In the generalizability evaluation, FSI was considered to have less of a priority. In correlation analyses, WC, systolic blood pressure, diastolic blood pressure, FSG, TG, total cholesterol, ALT, AST, $\gamma$-GT, and blood urea nitrogen were found representative (Figure S2). In a term of accuracy, height, blood urea nitrogen, creatinine, urinary protein, urinary glucose, and urinary $\mathrm{pH}$ were excluded. Finally, age, sex, WC, systolic blood pressure, FSI, TG, and ALT were selected and enrolled for multivariable analysis.

\section{Identification of independently indicative factors for NAFLD and development of K-NAFLD score}

In multivariable analysis, all factors were found significantly and independently indicative of NAFLD, except for age (OR, 0.996; 95\% CI, 0.987-1.005; P=0.425; Table S1). After excluding age from the model, all 6 factors revealed significance and independence, including sex (OR, 2.492; 95\% CI, 1.921-3.233; $\mathrm{P}<0.001)$, WC (OR, 1.093; $95 \%$ CI, 1.077-1.110; $\mathrm{P}<0.001)$, systolic blood pressure (OR, 1.033 ; 95\% CI, 1.025-1.040; $\mathrm{P}<0.001)$, FSI (OR, 1.032; 95\% CI, 1.026-1.038; $\mathrm{P}<0.001)$, TG (OR, 1.007; $95 \%$ CI, 1.006-1.009; $\mathrm{P}<0.001)$, and ALT (OR, 1.110; 95\% CI, 1.097-1.124; $\mathrm{P}<0.001)$, which constituted the KNHANES NAFLD (K-NAFLD) score (Table 3). Among the covariates, only FSG was missing in 10 participants. According to the intercept and estimate values, the score was formed as follows: $0.913 \times$ sex $(2$, if female; 1 , if male $)+0.089 \times$ WC $+0.032 \times$ (systolic blood pressure + FSG $)+$ TG $\times 0.007+$ ALT $\times 0.105-20.929$. The mean K-NAFLD scores for NAFLD and no NAFLD were 1.118 (95\% CI, 0.884-1.351) and -3.349 (95\% CI, -3.414 to -3.285$)$, respectively. Therefore, the K-NAFLD score $<-3.285$ and $>0.884$ were 
Table 2 Univariable analysis of factors involved in the health examination

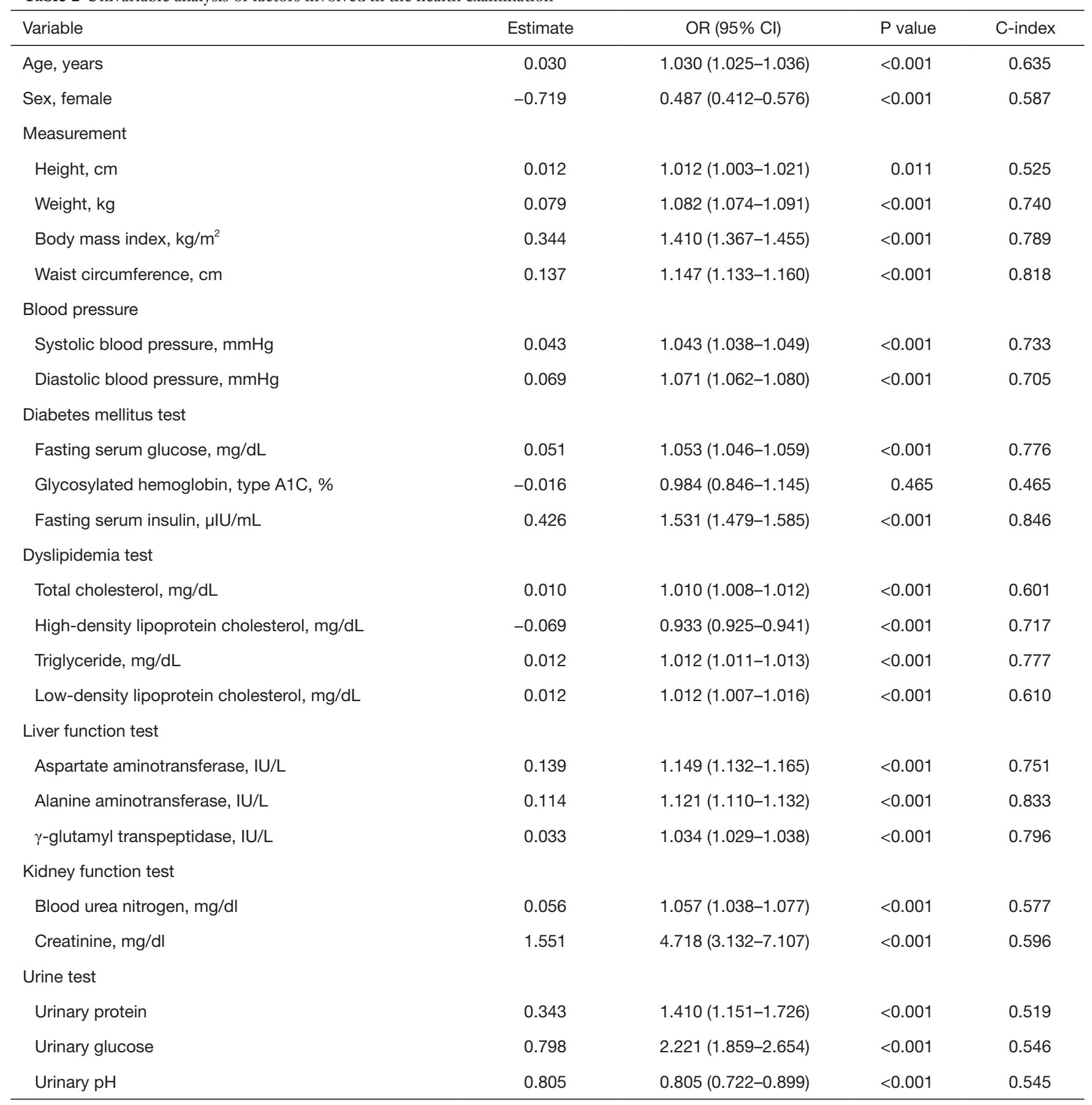

OR calculated by univariable logistic regression model. All variables are continuous, except for sex. OR, odd ratio; $\mathrm{Cl}$, confidence interval. 
Table 3 Multivariable analysis-derived model for prediction of K-NAFLD

\begin{tabular}{lccc}
\hline Covariate & Estimate & OR (95\% Cl) & P value \\
\hline Intercept & -20.929 & & $<.001$ \\
Sex, female & 0.913 & $2.492(1.921-3.233)$ & $<0.001$ \\
Waist circumference, $\mathrm{cm}$ & 0.089 & $1.093(1.077-1.110)$ & $<0.001$ \\
Systolic blood pressure, $\mathrm{mmHg}$ & 0.032 & $1.033(1.025-1.040)$ & $<0.001$ \\
Fasting serum glucose, $\mathrm{mg} / \mathrm{dL}$ & 0.032 & $1.032(1.026-1.038)$ & $<0.001$ \\
Triglyceride, $\mathrm{mg} / \mathrm{dL}$ & 0.007 & $1.007(1.006-1.009)$ & $<0.001$ \\
Alanine aminotransferase, IU/L & 0.105 & $1.110(1.097-1.124)$ & $<0.001$ \\
\hline
\end{tabular}

Variables were tested by the logistic regression. All variables were continuous, except for sex. K-NAFLD, Korea National Health and Nutrition Examination Survey-derived nonalcoholic fatty liver disease; OR, odd ratio; CI, confidence interval.

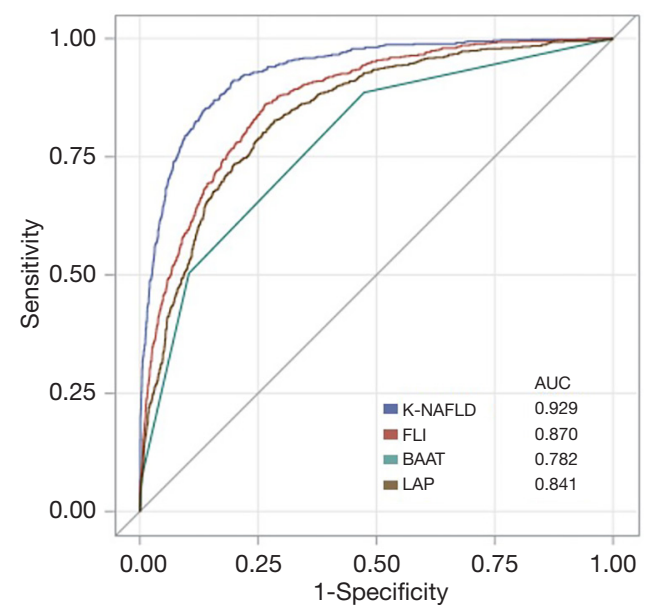

Figure 1 Receiver operating curves evaluating indicative performance of K-NAFLD, FLI, BAAT, LAP, and HSI for NAFLD. K-NAFLD, Korea National Health and Nutrition Examination Survey-derived nonalcoholic fatty liver disease; BAAT, body mass index, age, alanine aminotransferase, and triglycerides; LAP, lipid accumulation product; HSI, hepatic steatosis index; NAFLD, nonalcoholic fatty liver disease.

set as cut-off values for no NAFLD and NAFLD.

\section{Evaluation of the K-NAFLD score and comparison of the NAFLD-related scoring systems}

The ROC curves of K-NAFLD, FLI, BAAT, and LAP for diagnosis of NAFLD indicated to be excellent since these systems were developed with intent to diagnose NAFLD (Figure 1). Among them, the K-NAFLD score was found most accurate (AUC $=0.929$ ), followed by FLI (AUC
$=0.870)$, LAP $($ AUC $=0.841)$, and BAAT $(\mathrm{AUC}=0.782)$. The ROC contrast estimation and testing showed that $\mathrm{K}-\mathrm{NAFLD}$ score is significantly different compared to FLI (estimate, $-0.051 ; \mathrm{P}<0.001$ ), LAP (estimate, -0.080 ; $\mathrm{P}<0.001$ ), BAAT (estimate, $-0.141 ; \mathrm{P}<0.001$ ), and HSI (estimate, $-0.282 ; \mathrm{P}<0.001)$. The percent concordant for the K-NAFLD was 92.9, which was higher than FLI (87.0), BAAT (65.9), LAP (84.1), and HSI (66.7; Table S2). In addition, the positive predictive value and negative predictive value for K-NAFLD, FLI, and HSI were 0.990 and $0.860,0.935$ and 0.723 , and 0.785 and 0.182 , respectively. Subsequently, we sought to confirm the extent of correlations among the scoring systems. As described in the scatter correlation matrix, K-NAFLD score was highly correlated with the NAFLD liver fat score $(r=0.730)$, FLI $(r=0.789)$, BAAT score $(r=0.630)$, and LAP (r=0.672; Figure 2). Furthermore, the derived score was estimative of NAFLD in both BMI $<25 \mathrm{~kg} / \mathrm{m}^{2}$ (OR, 2.712; 95\% CI, 2.445-3.008; $\mathrm{P}<0.001$ ) and $\mathrm{BMI}$ $\geq 25 \mathrm{~kg} / \mathrm{m}^{2}$ (OR, 2.681; 95\% CI, 2.374-3.027; $\mathrm{P}<0.001$ ) subgroups (Table $\mathrm{S} 3$ ).

\section{Impact of $K-N A F L D$ score on metabolic syndrome, metabolic risk factors, obesity, and HOMA-IR}

To confirm potential applicability, we have testified whether the K-NAFLD score is informative of the metabolic risks by multivariable logistic regression adjusted for age, household income, smoking, and exercise (Table 4). The K-NAFLD score was highly estimative of metabolic syndrome (OR, 1.708; 95\% CI, 1.623-1.797; P<0.001), WC (OR, 1.510; 95\% CI, 1.440-1.584; $\mathrm{P}<0.001$ ), blood pressure (OR, 1.311; 95\% CI, 1.266-1.357; $\mathrm{P}<0.001)$, TG (OR, 


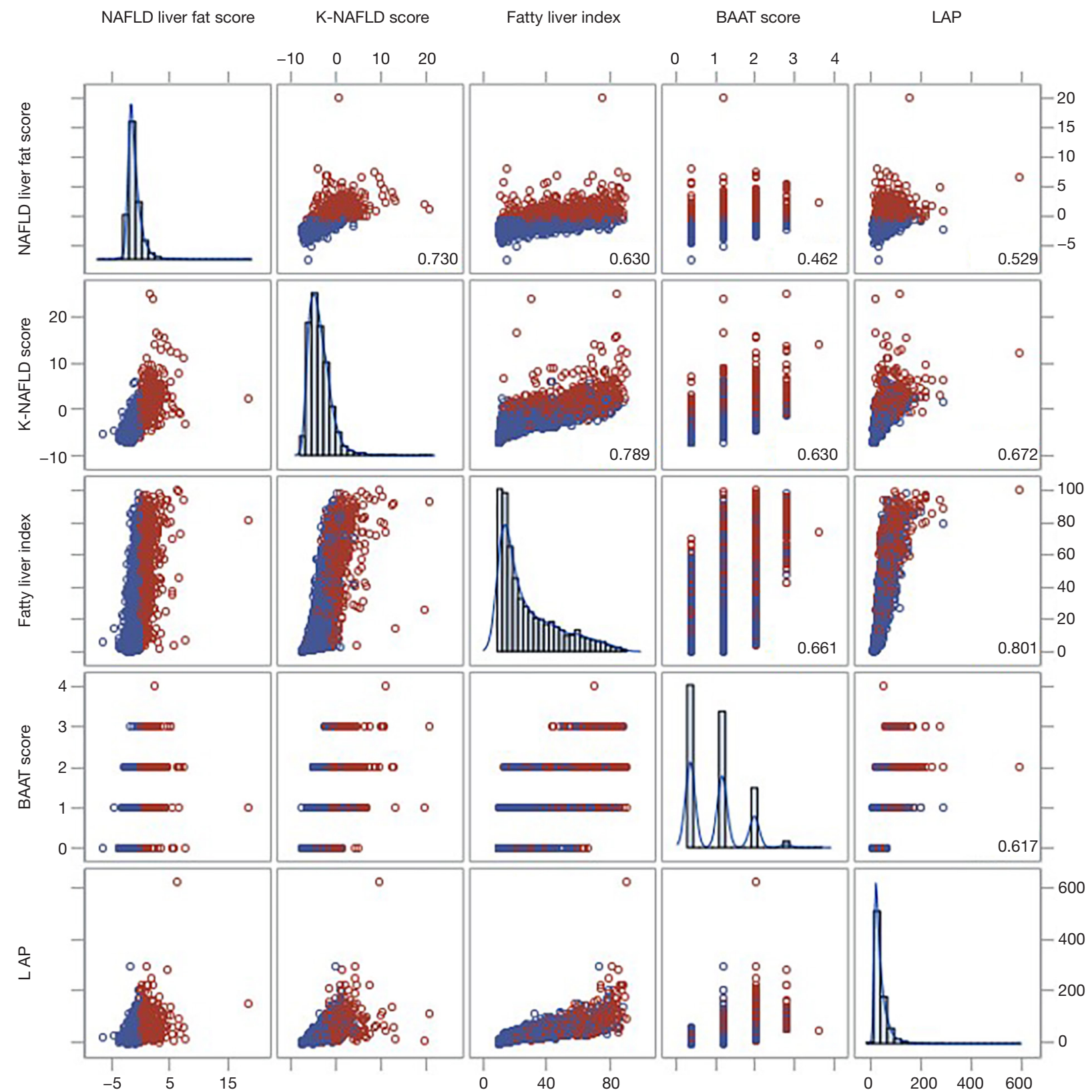

Figure 2 Scatter matrix for evaluation of correlations between NAFLD liver fat score, K-NAFLD score, FLI, BAAT, LAP, and HSI. Each scatter represents an individual. The extents of correlations are evaluated with $r$ values calculated by the Pearson's correlation test, which were presented in the lower right corner of the graphs. P values are presented in the upper right corner of the graphs. Red and blue scatters are the NAFLD and non-NAFLD cases, respectively. K-NAFLD, Korea National Health and Nutrition Examination Survey-derived nonalcoholic fatty liver disease; FLI, fatty liver index; BAAT, body mass index, age, alanine aminotransferase, and triglycerides; LAP, lipid accumulation product; HSI, hepatic steatosis index; NAFLD, nonalcoholic fatty liver disease.

$1.600 ; 95 \%$ CI, 1.533-1.669; $\mathrm{P}<0.001)$, HDL cholesterol (OR, 1.178; 95\% CI, 1.144-1.214; $\mathrm{P}<0.001$ ), and FSG (OR, 1.507; 95\% CI, 1.446-1.571; $\mathrm{P}<0.001$ ), obesity (OR,
1.824; 95\% CI, 1.740-1.913; $\mathrm{P}<0.001)$, and HOMA-IR (OR, 1.666; 95\% CI, 1.596-1.739; $\mathrm{P}<0.001$ ). Furthermore, in order to identify range-dependent outcomes, the 
Table 4 Performance of the K-NAFLD score for metabolic risk, obesity, and insulin resistance

\begin{tabular}{|c|c|c|c|c|}
\hline Variable & \multicolumn{2}{|c|}{ Mean K-NAFLD score } & OR (95\% Cl) & $P$ value \\
\hline Metabolic syndrome & 0.789 & -3.068 & $1.708(1.623-1.797)$ & $<0.001$ \\
\hline Waist circumference ${ }^{a}$ & 0.466 & -2.809 & $1.510(1.440-1.584)$ & $<0.001$ \\
\hline Blood pressure $^{b}$ & -0.905 & -3.210 & $1.311(1.266-1.357)$ & $<0.001$ \\
\hline HDL cholesterol $^{d}$ & -1.553 & -2.903 & $1.178(1.144-1.214)$ & $<0.001$ \\
\hline Fasting serum glucose $e^{e}$ & -0.038 & -3.138 & $1.507(1.446-1.571)$ & $<0.001$ \\
\hline Obesity, BMI $\geq 25 \mathrm{~kg} / \mathrm{m}^{2}$ & -0.361 & -3.398 & $1.824(1.740-1.913)$ & $<0.001$ \\
\hline HOMA-IR $\geq$ mean (2.533) & -0.765 & -3.398 & $1.666(1.596-1.739)$ & $<0.001$ \\
\hline
\end{tabular}

Variables are dichotomous, which were presented with OR calculated by the multivariable logistic regression model after adjustments for age, household income, smoking status, and exercise frequency. Metabolic syndrome was defined when three or more of the following criteria are met: ${ }^{a},>102 \mathrm{~cm}$ for men and $>88 \mathrm{~cm}$ for women; ${ }^{b}$, systolic blood pressure $\geq 130$ or diastolic blood pressure $\geq 85 \mathrm{mmHg}$; c, >150 mg/dL; ',$<40 \mathrm{mg} / \mathrm{dL}$ for men or $<50 \mathrm{mg} / \mathrm{dL}$ for women; and ${ }^{\mathrm{e}},>100 \mathrm{mg} / \mathrm{dL}$. K-NAFLD, Korea National Health and Nutrition Examination Survey-derived nonalcoholic fatty liver disease; OR, odd ratio; Cl, confidence interval; HDL, high-density lipoprotein; BMI, body mass index; HOMA-IR, homeostatic model assessment-insulin resistance.

Table 5 Adjusted means of metabolic risk factors according to the K-NAFLD score

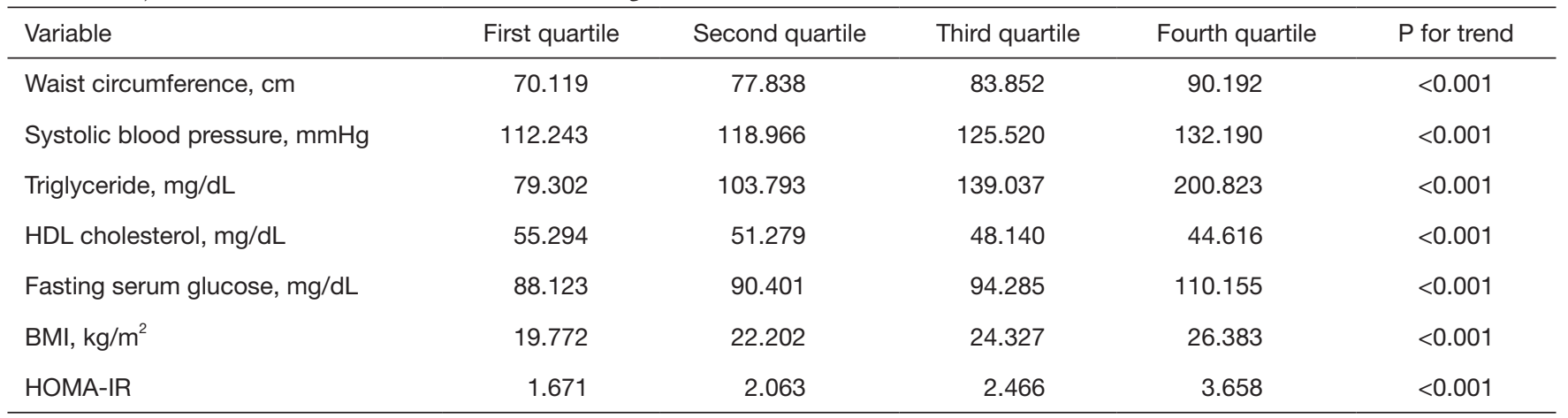

Mean values calculated after adjustments for age, household income, smoking status, and exercise frequency. K-NAFLD, Korea National Health and Nutrition Examination Survey-derived nonalcoholic fatty liver disease; HDL, high-density lipoprotein; BMI, body mass index; HOMA-IR, homeostatic model assessment-insulin resistance.

K-NAFLD score was stratified into 4 quartiles, of which all variables were in direct proportion to the K-NAFLD score (Table S4). In addition, the adjusted means of WC, systolic blood pressure, TG, HDL cholesterol, FSG, BMI, and HOMA-IR were in direct proportion to the quartiles of the K-NAFLD score (Table 5). As for mono-metabolic risk factors, including BMI ( $\mathrm{r}=0.622)$, systolic blood pressure $(\mathrm{r}=0.485)$, FSG $(\mathrm{r}=0.508)$, total cholesterol $(\mathrm{r}=0.317)$, the K-NAFLD score also revealed significant correlations with high concentrations within the $95 \%$ ellipse as confirmed by the Pearson's correlation test (Figure 3).

\section{Discussion}

NAFLD is nearly reaching pandemic proportion in parallel with an increase in the prevalence of obesity-related complications, which led to an increased prevalence of NASH carrying a high risk of liver-related morbidity and mortality in a real-world since reliable biomarkers are yet to be detected $(22,23)$.

In the present study, we derived K-NAFLD score indicative of NAFLD with no independent or external validation. From our point of view, benefits associated with deriving a model from the whole dataset are more effective 
A
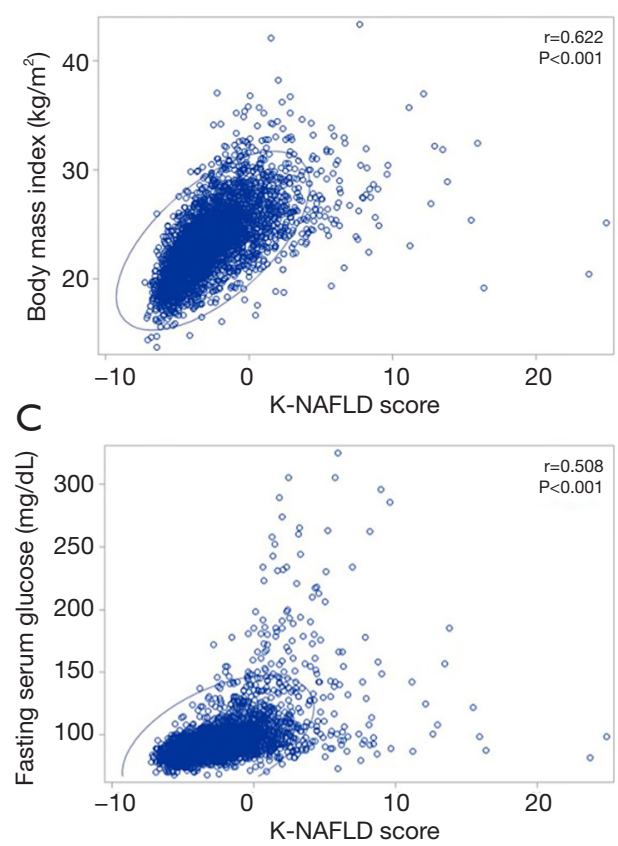

B
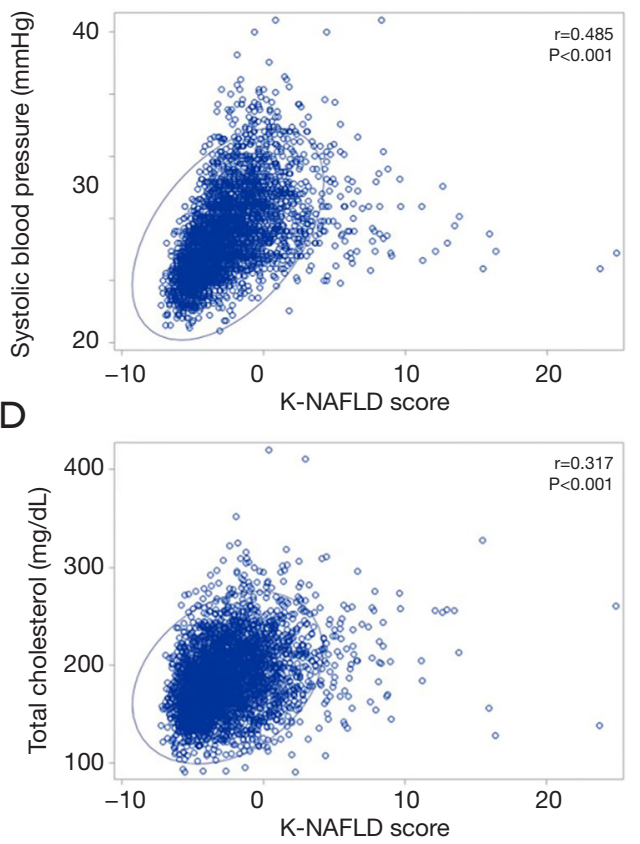

Figure 3 Correlations between metabolic risk factors and the K-NAFLD score. (A) Body mass index. (B) Systolic blood pressure. (C) Fasting serum glucose. (D) Total cholesterol. All values were calculated using the Pearson's correlation test. The ellipse shows the distribution of $95 \%$ cases. K-NAFLD, KNHANES NAFLD.

in terms of generalization and comprehensiveness compared to dividing the dataset into training and validation groups according to the characteristics of the dataset that is nationally representative of Korean citizens. Therefore, external validation is necessary for the K-NAFLD score to be applied in other countries.

There were a number of attempts with intent to predict disease progression to steatosis, such as BAAT and HSI (24). It is important to note that stratification of individuals at risk for advanced fibrosis is crucial to improve liver-related morbidity and mortality. However, it is also important to estimate those at increased risk for early NAFLD since it significantly effects economic burden. Furthermore, unlike previous studies developing predictive tools for NAFLD from Western countries, the study population had a mean BMI of 23.5 , despite it refers to overweight according to the Asian criteria (25). In fact, there was no study non-invasively evaluating NAFLD with this much low BMI. In addition, there is yet a suitable model for the Eastern population considering influence of ethnicity on prediction of NAFLD (26). Hence, we sought to develop a new continuous scoring system estimative of metabolic risk for general population in real-world using a
Korean representative dataset.

In the present study, FSG was selected alternatives to FSI in quality control for K-NAFLD score since the aim of this study was to define a simple, easily applied, and applicable to general cohort studies. In addition, FSI is absent in most large datasets, including the nationwide longitudinal cohort of health examination data of South Korea and the surveillance, epidemiology, and end results program of the United States, indicating limited generalizability. Despite an alternative covariate selection, the K-NAFLD score demonstrated 0.929 of AUC and 0.948 of true positive rate. From our point of view, the correlation $(r=0.265)$ between FSG and FSI may have led FSG to reflect individualized FSI levels. Furthermore, glycosylated hemoglobin type A1C was found not to be significantly associated with the presence of NAFLD. A previous study from China has reported that glycosylated hemoglobin level is not significantly different between healthy and mild NAFLD groups, but it significantly increased in moderate and severe NAFLD groups, suggesting that the glycosylated hemoglobin is still important in terms of NAFLD and needs to be considered especially when evaluating the severity of NAFLD (27).

The K-NAFLD score included sex, WC, systolic blood 
pressure, and TG, which are not involved in the NAFLD liver fat score. The proportion of female participants was $62.5 \%$, which is $9.5 \%$ higher than the NAFLD liver fat score study. The impact of sex difference on the risk of NAFLD among general population is conflicting. A study from Dallas demonstrated that white men had higher risk of NAFLD compared to women (28). On the contrary, a study from the University of North Carolina showed that women have higher prevalence of NAFLD in a setting of high visceral fat (29). From our point of view, we hypothesize that either of the following may be responsible for higher risk of NAFLD in female. First is the lifestyle disparities that physical activities might be less frequent in women compared to men or the extent of fat consumption may be relatively high among women as suggested by the study from North Carolina. However, the prevalence of NAFLD was dependent to the reproductive age among women that those within the reproductive age showed a low prevalence of NAFLD, thus interaction between sex and other covariates needs to be considered when interpreting structure of the derived model. Another concern is that sexbased difference in body fat distribution may be responsible for high prevalence of NAFLD in Eastern women population. To ascertain our hypotheses, future studies with behavioral information analysis are necessary.

Another important concern is that why the K-NAFLD score is well correlated with all scoring systems, including the NAFLD liver fat score, FLI, BAAT score, LAP, and HSI despite the differences in covariate distribution, but the K-NAFLD score was most highly correlated with the NAFLD liver fat score. In our perspective, the FLI involved more than one covariate within a section, such as BMI and WC, that there may be a duplication-related error due to significant correlation despite a complex equation that is incalculable by hand. As for BAAT, this system may have insufficiently reflected NAFLD due to missing covariate reflecting diabetes mellitus test and blood pressure sections. In addition, LAP is even more simplified as this system was not developed with intent to diagnose NAFLD. Therefore, we suggest future studies to comprehensively identify all potentially NAFLD-reflective covariates and include for prediction of NAFLD for improvement in terms of discrimination and accuracy.

To date, most non-invasive methods relied on 2 approaches, including a biological approach based on serologic biomarkers and a physical approach based on liver stiffness. These approaches are still complementary despite no strict liver-specificity and less accuracy compared to liver biopsy (30). The derived K-NAFLD score is highly easy to implement and has potential to be applied as a baseline approach for stratification of individuals who to undergo screening for NAFLD. Furthermore, the K-NAFLD score has another potential applicability that this continuous score may be informative for advanced fibrosis or steatosis, which awaits future studies to confirm.

Despite accurate and simple approach to estimate NAFLD along with metabolic risk, the K-NAFLD score has underlying limitations. First, no independent validation was performed since this score showed high correlations with already independently validated scoring systems. In addition, we are planning to validate applicability of the K-NAFLD score for estimation of cardiometabolic risk, as well as other outcomes in near future. Second, no liver biopsy nor imaging-based diagnostic information is involved, but applied the NAFLD liver fat score criteria for diagnosis of NAFLD because the NAFLD liver fat score was generated on the basis of the liver fat measurement by histology and proton magnetic resonance spectroscopy. Another concern is non-inclusion of potential predictive factors for NAFLD, such as dietary habits and regularity of sleeping, which awaits future studies to evaluate in the future.

In conclusion, the K-NAFLD score, composed of sex, WC, systolic blood pressure, FSG, TG, and ALT, is significantly indicative of NAFLD, and shows significantly estimative impact on metabolic syndrome, metabolic risk factors, obesity, and insulin resistance. Future studies comparing the derived score with a standard diagnostic test-proven data, such as ultrasonography of the liver, magnetic resonance proton density fat fraction in the liver, FibroScan with controlled attenuation parameter, and liver biopsy, are necessary for the derived score to be implemented.

\section{Acknowledgments}

Funding: None.

\section{Footnote}

Reporting Checklist: The authors present the study in accordance with the STROBE reporting checklist. available at: http://dx.doi.org/10.21037/atm-20-2951

Peer Review File: Available at http://dx.doi.org/10.21037/ atm-20-2951 
Conflicts of Interest: All authors have completed the ICMJE uniform disclosure form (available at: http://dx.doi. org/10.21037/atm-20-2951). KK, SMK, and SJ received a grant from the BK21-plus education program provided by the National Research Foundation of Korea. The other authors have no conflicts of interest to declare.

Ethical Statement: The authors are accountable for all aspects of the work in ensuring that questions related to the accuracy or integrity of any part of the work are appropriately investigated and resolved. The study was conducted in accordance with the Declaration of Helsinki (as revised in 2013). The institutional review board of the Korea Center for Disease Control and Prevention reviewed and approved the KNHANES (2008-04EXP-01-C, 2009-01CON-03-2C, 2010-02CON-21-C; https://www. cdc.go.kr).

Open Access Statement: This is an Open Access article distributed in accordance with the Creative Commons Attribution-NonCommercial-NoDerivs 4.0 International License (CC BY-NC-ND 4.0), which permits the noncommercial replication and distribution of the article with the strict proviso that no changes or edits are made and the original work is properly cited (including links to both the formal publication through the relevant DOI and the license). See: https://creativecommons.org/licenses/by-nc-nd/4.0/.

\section{References}

1. Budd J, Cusi K. Non-Alcoholic Fatty Liver Disease: What Does the Primary Care Physician Need to Know? Am J Med 2020;133:536-43.

2. Sayiner M, Koenig A, Henry L, et al. Epidemiology of Nonalcoholic Fatty Liver Disease and Nonalcoholic Steatohepatitis in the United States and the Rest of the World. Clin Liver Dis 2016;20:205-14.

3. Estes C, Razavi H, Loomba R, et al. Modeling the epidemic of nonalcoholic fatty liver disease demonstrates an exponential increase in burden of disease. Hepatology 2018;67:123-33.

4. Huang TD, Behary J, Zekry A. Non-alcoholic fatty liver disease (NAFLD): a review of epidemiology, risk factors, diagnosis and management. Intern Med J 2019. doi: 10.1111/imj.14709.

5. Mittal S, El-Serag HB, Sada YH, et al. Hepatocellular Carcinoma in the Absence of Cirrhosis in United States Veterans is Associated With Nonalcoholic Fatty Liver
Disease. Clin Gastroenterol Hepatol 2016;14:124-31.e1.

6. Piscaglia F, Svegliati-Baroni G, Barchetti A, et al. Clinical patterns of hepatocellular carcinoma in nonalcoholic fatty liver disease: A multicenter prospective study. Hepatology 2016;63:827-38.

7. Grundy SM, Cleeman JI, Daniels SR, et al. Diagnosis and management of the metabolic syndrome: an American Heart Association/National Heart, Lung, and Blood Institute scientific statement: Executive Summary. Crit Pathw Cardiol 2005;4:198-203.

8. Boza C, Riquelme A, Ibañez L, et al. Predictors of nonalcoholic steatohepatitis (NASH) in obese patients undergoing gastric bypass. Obes Surg 2005;15:1148-53.

9. Dietrich P, Hellerbrand C. Non-alcoholic fatty liver disease, obesity and the metabolic syndrome. Best Pract Res Clin Gastroenterol 2014;28:637-53.

10. Bedossa P. Diagnosis of non-alcoholic fatty liver disease/ non-alcoholic steatohepatitis: Why liver biopsy is essential. Liver Int 2018;38 Suppl 1:64-6.

11. Castera L. Diagnosis of non-alcoholic fatty liver disease/ non-alcoholic steatohepatitis: Non-invasive tests are enough. Liver Int 2018;38 Suppl 1:67-70.

12. Ginès P, Graupera I, Lammert F, et al. Screening for liver fibrosis in the general population: a call for action. Lancet Gastroenterol Hepatol 2016;1:256-60.

13. Kotronen A, Peltonen M, Hakkarainen A, et al. Prediction of non-alcoholic fatty liver disease and liver fat using metabolic and genetic factors. Gastroenterology 2009;137:865-72.

14. Bedogni G, Bellentani S, Miglioli L, et al. The Fatty Liver Index: a simple and accurate predictor of hepatic steatosis in the general population. BMC Gastroenterol 2006;6:33.

15. Ratziu V, Giral P, Charlotte F, et al. Liver fibrosis in overweight patients. Gastroenterology 2000;118:1117-23.

16. Kahn HS. The "lipid accumulation product" performs better than the body mass index for recognizing cardiovascular risk: a population-based comparison. BMC Cardiovasc Disord 2005;5:26.

17. Lee JH, Kim D, Kim HJ, et al. Hepatic steatosis index: a simple screening tool reflecting nonalcoholic fatty liver disease. Dig Liver Dis 2010;42:503-8.

18. Kweon S, Kim Y, Jang MJ, et al. Data resource profile: the Korea National Health and Nutrition Examination Survey (KNHANES). Int J Epidemiol 2014;43:69-77.

19. Pan WH, Yeh WT. How to define obesity? Evidencebased multiple action points for public awareness, screening, and treatment: an extension of Asian-Pacific recommendations. Asia Pac J Clin Nutr 2008;17:370-4. 
20. Huang PL. A comprehensive definition for metabolic syndrome. Dis Model Mech 2009;2:231-7.

21. Matthews DR, Hosker JP, Rudenski AS, et al. Homeostasis model assessment: insulin resistance and beta-cell function from fasting plasma glucose and insulin concentrations in man. Diabetologia 1985;28:412-9.

22. Araújo AR, Rosso N, Bedogni G, et al. Global epidemiology of non-alcoholic fatty liver disease/nonalcoholic steatohepatitis: What we need in the future. Liver Int 2018;38 Suppl 1:47-51.

23. Papatheodoridi M, Cholongitas E. Diagnosis of Nonalcoholic Fatty Liver Disease (NAFLD): Current Concepts. Curr Pharm Des 2018;24:4574-86.

24. Lee J, Ha J, Jo K, et al. Male-specific association between subclinical hypothyroidism and the risk of non-alcoholic fatty liver disease estimated by hepatic steatosis index: Korea National Health and Nutrition Examination Survey 2013 to 2015. Sci Rep 2018;8:15145.

25. WHO Expert Consultation. Appropriate body-mass index for Asian populations and its implications for policy and

Cite this article as: Jeong S, Kim K, Chang J, Choi S, Kim SM, Son JS, Lee G, Kim W, Park SM. Development of a simple nonalcoholic fatty liver disease scoring system indicative of metabolic risks and insulin resistance. Ann Transl Med 2020;8(21):1414. doi: 10.21037/atm-20-2951 intervention strategies. Lancet 2004;363:157-63.

26. Xia MF, Yki-Järvinen H, Bian H, et al. Influence of Ethnicity on the Accuracy of Non-Invasive Scores Predicting Non-Alcoholic Fatty Liver Disease. PLoS One 2016;11:e0160526.

27. Cai J, Zhang Z, Liu J, et al. Correlation between serum 25-OH vitamin D expression and non-alcoholic fatty liver disease. Exp Ther Med 2020;19:1681-6.

28. Browning JD, Szczepaniak LS, Dobbins R, et al. Prevalence of hepatic steatosis in an urban population in the United States: impact of ethnicity. Hepatology 2004;40:1387-95.

29. Graff M, North KE, Franceschini N, et al. PNPLA3 gene-by-visceral adipose tissue volume interaction and the pathogenesis of fatty liver disease: the NHLBI family heart study. Int J Obes (Lond) 2013;37:432-8.

30. Castera L, Friedrich-Rust M, Loomba R. Noninvasive Assessment of Liver Disease in Patients With Nonalcoholic Fatty Liver Disease. Gastroenterology 2019;156:1264-1281.e4. 